Original article

\title{
Prevalence and pattern of substance use among sandstone mine workers in Rajasthan, India
}

\author{
Absar Ahmad $^{\mathrm{a}, *}$, Ishrat Rahman ${ }^{\mathrm{b}}$, Manoj Alagarajan ${ }^{\mathrm{c}}$ \\ ${ }^{\text {a }}$ Department of Community Medicine, Career Institute of Medical Sciences and Hospital, Lucknow, India \\ ${ }^{\mathrm{b}}$ Basic Dental Sciences, College of Dentistry, Princess Nourah bint Abdulrahman University, Riyadh, Kingdom of Saudi Arabia \\ ${ }^{\mathrm{c}}$ Department of Development Studies, International Institute for Population Sciences, Mumbai, India
}

\section{A R T I C L E I N F O}

\section{Keywords:}

Mineworkers

Pattern

Prevalence

Substance use

Rajasthan

India

\begin{abstract}
A B S T R A C T
Background: The workplace is one of the important sites where substance use problems occur; very few studies identify the relationship between work and substance use in India. The current research was performed in a rural area where mining mostly occurs, to study the association of some of the risk factors of substance use.

Objective: This study examines the prevalence and pattern of substance use among mineworkers in Karauli district of Rajasthan, India.

Methods: The cross-sectional study included individuals over the age of 15 years, 218 mineworkers and 203 nonmine workers used for comparison purpose. Ten villages were selected using probability proportional to size sampling technique and participants were recruited using non-random sampling from each village from May 2014 to September 2014. Descriptive and analytical methods were used to estimate the prevalence and to identify the associated risk factors.

Results: Logistic regression analysis showed that mineworkers were at a higher risk of substance abuse as compared to the non-mine workers. Prevalence of consumption of khaini, bidi, alcohol, and gutkha was higher among mineworkers while the use of hookah smoking was among non-mine workers. Association analyses identified that factors such as age at starting work, awareness of work, associated risks, injury at a workplace, respiratory illness, periodontal problems or gum pain, and being in debt were significantly associated with uses of such hazardous substances among the mineworkers.

Conclusion: This may be the first study reporting on substance use among mine workers. The prevalence of substance use among mineworkers in this study was higher than the comparison group. Moreover, it correlates with multiple health problems suggesting a need to promote specific interventions to prevent mine workers towards substance use.
\end{abstract}

\section{Introduction}

The workplace, in particular, the mining industry has one of the highest rates of substance use and their associated problems. ${ }^{1,2}$ Mining is a physically tedious job that steers workers to consume harmful substances like alcohol and tobacco, ${ }^{3}$ contributing to illness, workplace accidents and injuries. ${ }^{4}$ The factors providing a theoretical explanation for the high incidence of substance use at the workplace and playing a leading role in the promotion of substance use are, workplace culture, ${ }^{5}$ social influence ${ }^{4}$ and social availability of alcohol. ${ }^{6}$

Consistent with these theoretical models, literature shows that workers who are heavy drinkers are often found to be engaged in drinking alcohol with co-workers during work breaks and after work. ${ }^{7}$ Also, the link between social norms and the usage of harmful substances demonstrates that workers who spend a large portion of their time at the workplace are more susceptible to fall for substance abuse. ${ }^{6}$ Availability of alcohol at work and drinking with friends and coworkers was the strongest predictor of work-related drinking problems. $^{8}$

Despite the critical link between workplace and substance use, little is known regarding patterns of the consumption of hazardous substances among the mineworkers in India. Notably, studies on mineworkers show weekly alcohol consumption to be significantly higher among workers with a greater workload; those who mostly work underground, and those working as drillers or cart pushers. ${ }^{9}$ Although there is no data available on alcohol consumption at workplaces in India, studies are documenting a high prevalence (68\%) of drinking habits among the male industrial workers. ${ }^{10}$ Furthermore, very few of

\footnotetext{
* Corresponding author.

E-mail addresses: r.absar.ahmad@gmail.com (A. Ahmad), ishrat.rahman.ir@gmail.com (I. Rahman), alagarajan@iips.net (M. Alagarajan).
} 


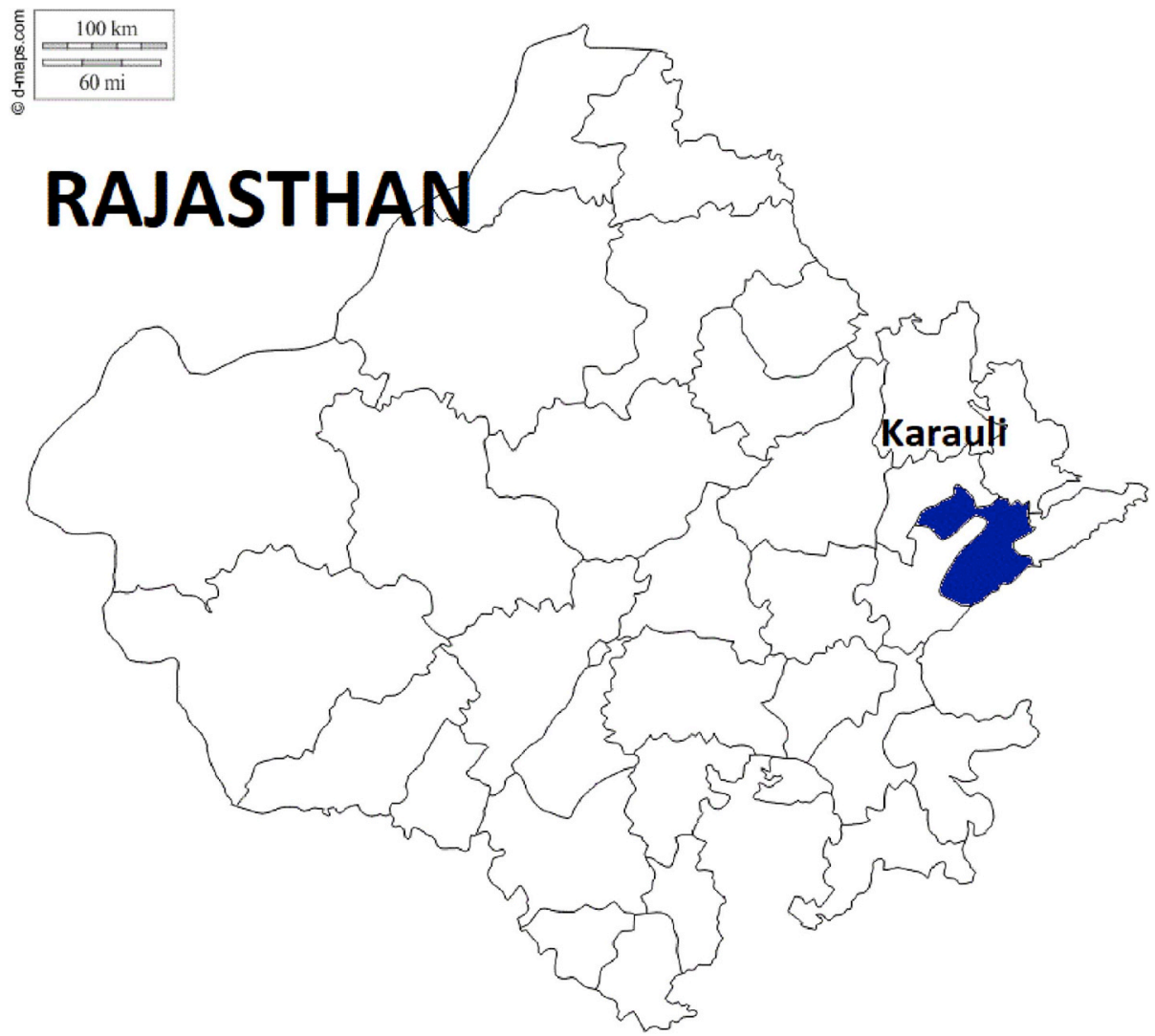

Fig. 1. Study area.

the studies that examined associated demographic and substance use factors have focused their research on mine workers, who have higher rates of substance abuse as compared to the general public. ${ }^{11}$ In this regards, this study was conducted to see the patterns of substance use among mine workers. The purpose of this study was to examine the demographic, substance use and the associated factors among mine workers. This information is essential for reducing the associated health risks and for better planning and execution of health programs regarding tobacco and alcohol prevention and treatment.

\section{Methods}

\subsection{Study design and population}

A cross-sectional survey was conducted in Karauli district (Fig. 1) of Rajasthan state in India during May to September 2014. The sample size for the study was determined using the formula $\mathrm{Z}^{2} \mathrm{pq} / \mathrm{d}^{2}$ ( $\mathrm{p}$ ' is the proportion of miners to total workers in Karauli block, ' $\mathrm{Z}$ ' is the type 1 error at $5 \%$, and' is the absolute error). Therefore, based on sample size calculations $\left(\mathrm{p}=0.15^{12}, \mathrm{Z}=1.96, \mathrm{~d}=0.05\right)$ the random and representative sample size required were 196 mine workers. However, interviews were conducted for 218 mine workers, and for comparison purpose 203 non-mine workers, who lived among the mine workers or in their close vicinity and similar socio-economic conditions. This group was comparable to the mineworker in all the variables except the risk of exposure.

\subsection{Selection of the study participants}

The current study adopted a two-stage sampling study design to increase the efficiency of the required number of respondents for interviews. In the first stage, ten villages were selected using probability proportional to population size (PPS) sampling. The village list was taken from primary census abstract and arranged according to the category of 'Other Workers'. A non-probability convenience sampling technique was used, due to a lack of availability of a registered list of mine workers. The mine workers and non-mine workers were selected at the concern of the researcher and others were not involved in the selection process. The study included all workers above 15 years of age. The workers were generally cooperative, and there were no refusals for taking part in the study.

\subsection{Survey instrument}

Demographic variables of interest included categorical age in years $(<30,31-40,46-60,60+)$, Monthly Income $(<4000,4001-7000,7000+)$ in INR, age in years at the time of starting work $(<15,16-30,30+)$, age at the time of beginning substance use, the caste of the worker (scheduled caste(SC), scheduled tribes(ST), Other backward class(OBC) and General), marital status (Single, Married, divorced/separated), years of schooling and occupational category (mineworkers and non- mineworkers), injury at workplace such as a fall from a rock, fall from height, struck or hit by objects are categorised in this variable identified in the form of yes and no, self-reported gum pain in last three months. Respiratory health status variable was constructed using four self-reported questions as regular cough, chest pain, shortness of breath, and wheezing in the chest.

Substance use behaviours of interest included use of Alcohol, Bidi (small hand-rolled cigarettes made of tobacco and wrapped in a tendu or temburni leaf), Gutkha (a sweetened mixture of chewing tobacco, betel nut, and palm nut) and Khaini (raw tobacco with slaked lime). Others substances were hookah (a single or multi-stemmed instrument for vaporising and smoking flavoured tobacco), Zarda (flavoured tobacco blending tobacco leaves, sweeteners and other compounds), Ganja (Cannabis), Cigarette and Supari (Betel net). Gutkha, Khaini and Zarda are the smokeless tobacco; these types of substances are consumed orally, not smoked and are well known to be equally harmful as 
smoking. ${ }^{13}$

Respondents were asked if they had used at least one of these substances in the last 30 days. We kept the time duration of 30 days to reduce recall bias, allowing respondents to easily and accurately recall exposure. Therefore, we assume recall bias or response bias to be negligible. Respondents indicating the use of a substance were given a score of one (1), and those who had not used a substance were scored as zero (0).

\subsection{Statistical analysis}

Data were entered in Epi Info software, and data analysis performed using Statistical Package for Social.

Sciences (SPSS, version 21). Bivariate analysis for categorical data was carried out using $\chi 2$ test.

Logistic regression analysis was performed to explore independent variables that were predictors of substance use. The probability of significance was set at $5 \%$.

\section{Ethical approval}

Approval for this study was granted from the Students research committee of International Institute for Population Sciences, Mumbai, India. Informed consent was obtained from all the study participants after thoroughly explaining the nature and purpose of the study.

\section{Results}

Table 1 shows the prevalence of use of each substance among mineworkers and non-mineworkers with age at first use indicated. Approximately $26 \%$ of mineworkers and $10 \%$ of non-mine workers had consumed alcohol in the past 30 days, $45 \%$ of mineworkers and $37 \%$ of non- mine workers had smoked bidi in the past 30 days, $23 \%$ of mineworkers and $16 \%$ of non- mine workers had chewed gutkha in the past 30 days, $46 \%$ and $24 \%$ of mineworkers and non-mine workers reported using khaini in the past 30 days. Around 9\% of non-mine workers smoked hookah; however, this figure was only $3 \%$ among mineworkers. Overall, the prevalence of substance use was significantly higher among the mineworkers (86\%) than among the non- mineworkers (69\%). The age at the onset of use of alcohol among mineworkers was 12 years, whereas, among non-mineworkers, it was 10 years. Similarly, the age at the onset of use of bidi, khaini, gutkha and hookah among mineworkers were 10,10,10 and 15 respectively. These ages among non- mine workers were 5, 13, 10 and 5 respectively.

Table 2 shows the worker's age at which they started work ( $p=0.001)$, awareness of risk in job $(p=0.000)$, injury at workplace $(\mathrm{p}=0.002)$, self-reported periodontal problems or gum pain $(\mathrm{p}=0.030)$, self -reported respiratory illness (0.002) and debt $(\mathrm{p}=0.024)$ were significantly associated with uses of substance. Although age, education, marital status and household income also mattered, none of these factors was significantly associated with the use of a substance in this study.

The logistic regression analysis (Table 3) shows that mineworkers were at a higher risk of substance abuse as compared to the non-mine workers (AOR 2.02(95\% CI 1.012; 4.034). The independent risk factors for substance use among the workers were exclusive to those who started work in the age group 16-30 years (AOR 0.339 (95\% CI 0.186; $0.619)$ compared to the reference age group of $<15$ years.

\section{Discussion}

Over 2.5 million workers in the Rajasthan state are engaged in mining, ${ }^{14}$ and the majority of these mines are unorganised. ${ }^{15}$ In most of the unorganised sector, the occupation exposes workers to both physical $^{16}$ and psychosocial risks ${ }^{16,17}$ and carries higher risk and exposure to toxic substances. ${ }^{18}$

In India, there is currently very little data identifying the prevalence of substance use; thus, it is still an unexplored field. The present study shows that the overall prevalence of substance use was higher among the mine workers (87\%) compared to the non-mine workers (70\%). In a similar type of study done in India on 510 stone mine workers, $94 \%$ of workers had the habit of substance use. ${ }^{19}$

The prevalence of use of individual substances was also higher among mineworkers in comparison to non-mine workers for khaini, bidi, alcohol and gutkha except hookah. Previously published similar studies conducted in India, report only about the use of smoking and alcohol consumption among mineworkers. The use of other substances like smokeless tobacco had not been addressed. However, the present study highlights the use of smokeless tobacco like khaini and gutkha in mineworkers.

In this study, we identified the prevalence of bidi smoking to be $45 \%$ whereas in other studies it was found to be 90 and 31\% among mineworkers by Yadav et al. $(2011)^{20}$ and Mehta(2010) ${ }^{21}$ respectively. Bidis produce equal or higher levels of nicotine, tar and other toxic chemicals, when compared to cigarettes and thus can lead to vascular disease, lung disease and cancers, just like other smoking products. ${ }^{22}$

Khaini (46\%) was found to be equally popular as bidi in the present study. A study carried out by Mehta (2010) found that $82 \%$ of mineworkers use bidi. Mineworkers reported that during their tedious fieldwork, they use khaini or bidi to make their time more enjoyable and leisurely. A study conducted in India found the prevalence of use of khaini (31\%) and gutkha (56\%) among factory employees; this was higher than the general population. ${ }^{23}$ Bidi is the most widely used substance among both the groups in Karauli. Both bidi and smokeless tobacco are cheap, least taxed, and the most common tobacco products in India. ${ }^{21} \mathrm{~A}$ recent national study found that among men, khaini (17.9\%) and bidi $(14.0 \%)$ were the most commonly used tobacco products in India. Khaini (13.5\%) and bidi (9.3\%) were also the most prevalent tobacco products in rural areas. ${ }^{24}$

Twenty-six (26) per cent of mineworkers consume alcohol. However, when comparing previously published studies, the prevalence of alcohol use in mineworkers was inconstant. A study done by Yadav

Table 1

Prevalence of substance use and age at initial use, among mine workers and non-mine workers.

Source: Primary survey.

\begin{tabular}{|c|c|c|c|c|c|c|}
\hline & Mine workers (218) & Minimum age at started & Non-mine workers (203) & Minimum age at started & $\mathrm{n}$ & Test of significance \\
\hline Alcohol & $26.60(58)$ & 12 & $10.30(21)$ & 10 & 95 & $\chi^{2}=23.35, p=0.000$ \\
\hline Bidi & $45.00(98)$ & 10 & $37.40(76)$ & 5 & 227 & $\chi^{2}=2.76, \mathrm{p}=0.252$ \\
\hline Khaini $^{\#}$ & $45.7(75)$ & 10 & $23.8(39)$ & 13 & 164 & $\chi^{2}=8.72, p=0.012$ \\
\hline Gutka $^{\#}$ & $22.90(50)$ & 10 & $16.30(33)$ & 10 & 96 & $\chi^{2}=10.88 \cdot p=0.004$ \\
\hline Hookah & $2.80(6)$ & 15 & $8.90(18)$ & 5 & 28 & $\chi^{2}=9.92, \mathrm{p}=0.007$ \\
\hline Cigarette & - & - & $0.50(1)$ & 20 & 2 & $\chi^{2}=1.41, \mathrm{p}=0.493$ \\
\hline Ganja & $0.50(1)$ & 30 & $1.00(2)$ & 25 & 3 & $\chi^{2}=1.52, p=0.466$ \\
\hline Zarda & $0.01(2)$ & 25 & - & - & 2 & $\chi^{2}=3.130, \mathrm{p}=0.209$ \\
\hline Supari & - & - & $0.5(1)$ & 27 & 1 & $\chi^{2}=1.752, p=0.416$ \\
\hline At least one & $85.8(187)$ & & $69.5(141)$ & & 328 & $\chi^{2}=19.13, p=0.000$ \\
\hline
\end{tabular}


Table 2

Prevalence of substance use with socio-demographic variables of the study population.

Source: Primary Survey.

\begin{tabular}{|c|c|c|c|c|}
\hline & & Mine workers 187 & Non-mine workers 141 & Test of significance \\
\hline \multirow[t]{4}{*}{ Age of respondents(in years), \% (n) } & $<30$ & $20.9(39)$ & $25.5(36)$ & \\
\hline & $31-45$ & $46.0(86)$ & $39.0(55)$ & $\chi 2=3.219, \mathrm{p}=0.359$ \\
\hline & $46-60$ & $31.0(58)$ & $19.9(28)$ & \\
\hline & $60+$ & $2.1(4)$ & $15.6(22)$ & \\
\hline \multirow[t]{3}{*}{ Education, \% (n) } & Illiterate & $43.3(81)$ & $36.2(51)$ & \\
\hline & Primary & $24.1(45)$ & $20.6(29)$ & $\chi 2=3.269, \mathrm{p}=0.195$ \\
\hline & High school and more & $32.6(61)$ & $43.3(61)$ & \\
\hline \multirow[t]{4}{*}{ Caste category, \% (n) } & SC & $52.4(98)$ & $14.2(20)$ & \\
\hline & ST & $10.2(19)$ & $7.1(10)$ & $\chi^{2}=.982, \mathrm{p}=0.806$ \\
\hline & OBC & $34.8(65)$ & $61.0(86)$ & \\
\hline & General & $2.7(5)$ & $17.7(25)$ & \\
\hline \multirow[t]{3}{*}{ Marital status, \% (n) } & Single & $5.3(10)$ & $15.6(22)$ & \\
\hline & Married & $92.0(172)$ & $75.2(106)$ & $\chi 2=5.151, \mathrm{p}=0.076$ \\
\hline & Divorced/Separated & $2.7(5)$ & $9.2(13)$ & \\
\hline \multirow[t]{2}{*}{ Household size, \% (n) } & $<5$ & $39.6(74)$ & $56.0(79)$ & $\chi^{2}=0.064, p=0.800$ \\
\hline & $\geq 5$ & $60.4(113)$ & $44.0(62)$ & \\
\hline \multirow[t]{3}{*}{ Monthly household income, \% (n) } & $<4000$ & $50.8(95)$ & $27.7(39)$ & \\
\hline & $4001-7000$ & $30.5(57)$ & $29.8(42)$ & $\chi 2=1.571, \mathrm{p}=0.456$ \\
\hline & $7001+$ & $18.7(35)$ & $42.6(60)$ & \\
\hline \multirow[t]{3}{*}{ Age at start job, \% (n) } & Less than 15 & $46.0(86)$ & $39.0(55)$ & \\
\hline & $16-30$ & $52.4(98)$ & $45.4(64)$ & $\chi 2=14.481, \mathrm{p}=0.001$ \\
\hline & $30+$ & $1.6(3)$ & $15.6(22)$ & \\
\hline \multirow[t]{2}{*}{ Awareness of risk in job, \% (n) } & No & $5.3(5)$ & $39.0(47)$ & \\
\hline & Yes & $94.7(182)$ & $61.0(94)$ & $\chi 2=13.987, p=0.001$ \\
\hline \multirow[t]{2}{*}{ Borrow loan, \% (n) } & No & $10.7(20)$ & $30.5(43)$ & \\
\hline & Yes & $89.3(167)$ & $69.5(98)$ & $\chi 2=5.081, p=0.024$ \\
\hline \multirow[t]{2}{*}{ Injury at workplace, $\%(n)$} & No & $34.8(65)$ & $71.6(101)$ & \\
\hline & Yes & $65.2(122)$ & $28.4(40)$ & $\chi 2=9.691, p=0.002$ \\
\hline \multirow[t]{2}{*}{ Respiratory Illness,\%(n) } & No & $15.0(28)$ & $70.9(100)$ & \\
\hline & Yes & $85.0(159)$ & $29.1(41)$ & $\chi^{2}=9.541, \mathrm{p}=0.002$ \\
\hline \multirow[t]{2}{*}{ Gum Pain,\%(n) } & No & $64.7(121)$ & $83.7(118)$ & \\
\hline & Yes & $35.3(66)$ & $16.3(23)$ & $\chi^{2}=4.718, p=0.030$ \\
\hline
\end{tabular}

et al. (2011) found the figure to be closer to 70\%. Another study found the prevalence to be $43 \% .{ }^{25}$ Mehta (2010) found the prevalence to be $22 \%$, which is comparable to the value presented in this study. However, another study found that $16 \%$ of marble mineworkers had alcohol addiction habits. ${ }^{26}$ One reason why alcohol consumption may be higher among mineworkers is that alcohol is readily available in villages and at mine sites. It is locally made and is relatively cheap, costing around Rs.35 (\$0.5) per bottle. The workers spend a considerable amount of their hard-earned money on smoking and drinking. Most of them used country liquor that is readily available in the village. Physically tedious work drives people to consume alcohol and tobacco ${ }^{25}$ Thus, the high prevalence of use of alcohol in mineworkers can be explained, by considering the nature of the work, which requires manual labour for long hours, and drinking alcohol might help them to reduce physical pain as well as facilitate in getting a sound sleep. Another reason reported by workers as a prevention strategy similar to the use of jaggery $^{20}$ that alcohol helped them in expelling out dust particle from the body through faeces. Initially, the mining contractor (thekedar) was freely providing alcohol. Many started taking alcohol with the belief that it will improve their work efficiency. Over time they became addicted to it, and the adverse effects of excessive drinking started to rear up, such as loss of consciousness, misbehaviour with the general public and domestic violence. Almost all mineworkers were involved in smoking and consuming alcohol. ${ }^{27}$ Thus it is understandable that workplace culture, acceptance of drinking and the availability of alcohol contribute to this alcohol-related phenomenon highlighted in this study. ${ }^{6}$ At mine sites, most of the workers were drinking or using some harmful substance. They tend to carry a full stock of these substances with them, for the whole day, to their work and also share it among their co-workers.

The current study revealed that about $9 \%$ of non-mine workers used hookah, whereas only $3 \%$ of the mineworkers use it. Nationally representative figures disclose the prevalence of hookah to be $1.1 \%$ among males and higher in the rural areas. In Rajasthan, its prevalence is about $2.7 \% .{ }^{24}$ Hookah consumption is a tradition in Rajasthan villages, but because of the complicated preparation process associated with it, this tradition is dying. Only a few of the well-off families use it nowadays. Mineworkers generally have a poor economic status and thus are not able to maintain this tradition.

People in the study area of Rajasthan do not consider these substances as harmful and even offer it to their guests as a gesture of hospitality. Women in these villages also consume bidi and khaini very frequently.

The bivariate analysis shows that risks in the job, injury at the workplace, reported respiratory illness and gum pain are significantly associated with substance use among workers. Previous studies have also shown that employees who are involved in jobs with higher physical risk report more substance use than employees working in relatively non-risky jobs. ${ }^{28}$ Thus it could be hypothesised that the working environment or reactions to the environment of high-risk positions may contribute to substance use problems. These positions may increase the opportunity for substance use, may provide a climate supportive of substance use, and may include job stressors that lead to increased use of this hazardous substances. ${ }^{29}$ Previous reports also show that injuries are higher among workers who use excessive substance. ${ }^{30}$

The current study found a higher incidence of reported respiratory illnesses among mineworkers using substances as compared to nonmine workers. Reports from population-based epidemiological studies found smoking to increase respiratory diseases in a diverse number of occupations. ${ }^{31}$ The present study also highlights a significant association between substance use and oral health. These findings are in agreement with previously published data among marble mine workers in India. ${ }^{26}$ Substance use is associated with increased pocket depth, loss of periodontal attachment, alveolar bone and a higher rate of tooth loss. ${ }^{32}$ Therefore, this could be the reason for having a higher incidence of periodontal diseases in high substance use, smoker's group. 
Table 3

Predictors of current use of substance among the workers from logistic regression.

Source: Primary survey.

\begin{tabular}{|c|c|c|}
\hline & & Adjusted Odds ratio (95\% C.I.) \\
\hline \multirow[t]{4}{*}{ Age of respondents } & $<30^{\circledR}$ & Ref \\
\hline & $31-45$ & $1.362(0.702-2.643)$ \\
\hline & $46-60$ & $1.392(0.619-3.129)$ \\
\hline & $60+$ & $1.485(0.481-4.583)$ \\
\hline \multirow[t]{3}{*}{ Education } & Illiterate $^{\circledast}$ & Ref \\
\hline & Primary & $1.872(0.895-3.918)$ \\
\hline & $\begin{array}{l}\text { High school and } \\
\text { more }\end{array}$ & $1.485(0.789-2.794)$ \\
\hline \multirow[t]{4}{*}{ Caste category } & $\mathrm{SC}^{\circledast}$ & Ref \\
\hline & ST & $1.108(0.427-2.875)$ \\
\hline & OBC & $1.537(0.818-2.888)$ \\
\hline & General & $2.05(0.739-5.686)$ \\
\hline \multirow[t]{4}{*}{ Marital status } & Single ${ }^{\circledR}$ & Ref \\
\hline & Married & $1.294(0.557-3.008)$ \\
\hline & Divorced/ & $0.532(0.156-1.816)$ \\
\hline & Separated & \\
\hline \multirow[t]{2}{*}{ Household size } & $<5^{\oplus}$ & Ref \\
\hline & $\geq 5$ & $0.635(0.365-1.105)$ \\
\hline \multirow{3}{*}{$\begin{array}{l}\text { Monthly household } \\
\text { income }\end{array}$} & $<4000^{\oplus}$ & Ref \\
\hline & $4001-7000$ & $1.194(0.617-2.311)$ \\
\hline & $7001+$ & $1.108(0.566-2.168)$ \\
\hline \multirow[t]{3}{*}{ Age at start job } & Less than $15^{\circledR}$ & Ref \\
\hline & $16-30$ & $0.339(0.186-0.619)^{* *}$ \\
\hline & $30+$ & $0.595(0.207-1.715)$ \\
\hline \multirow[t]{2}{*}{ Awareness of risk in job } & $\mathrm{No}^{\circledast}$ & Ref \\
\hline & Yes & $1.466(0.784-2.748)$ \\
\hline \multirow[t]{2}{*}{ Borrow loan } & $\mathrm{No}^{\oplus}$ & Ref \\
\hline & Yes & $1.183(0.649-2.156)$ \\
\hline \multirow[t]{2}{*}{ Injury at workplace } & $\mathrm{No}^{\oplus}$ & Ref \\
\hline & Yes & $1.337(0.764-2.34)$ \\
\hline \multirow[t]{2}{*}{ Respiratory Illness } & $\mathrm{No}^{\circledR}$ & Ref \\
\hline & Yes & $1.232(0.674-2.251)$ \\
\hline \multirow[t]{2}{*}{ Gum pain } & $\mathrm{No}^{\circledast}$ & Ref \\
\hline & Yes & $1.441(0.71-2.924)$ \\
\hline \multirow[t]{2}{*}{ Working status } & $\begin{array}{l}\text { Non-mine } \\
\text { workers }^{\circledR}\end{array}$ & Ref \\
\hline & Mine workers & $2.020(1.012-4.034) *$ \\
\hline
\end{tabular}

Notes: ${ }^{\circledR}$ Reference group.

$*$, ** Significant at 5 and $1 \%$ levels, respectively.

Multivariate analysis also shows that those who started to work in the age group of 16-30 years were less likely to use substances as compared to the age group under 15 years. Previous studies have shown that substance use peaks during late adolescence and young adulthood and decline thereafter. ${ }^{33}$ Early adolescent substance use also significantly increases the risk of lifelong substance use disorder. ${ }^{34}$ The teens that initiate substance use before the age of 14 years are at highest risk for substance dependence and have a $34 \%$ prevalence rate of lifetime substance use. ${ }^{35}$ Most of the mineworkers in the study area started by helping their fathers or other relatives in the mine work, and subsequently, they became a full-time mineworker. A large proportion, $43 \%$ of mineworkers, start their jobs at an age below 15 years. ${ }^{17}$ Therefore, it is highly conceivable that during this time the young workers are in contact with smokers and addicts, thus becoming addicted themselves.

Previous studies uncovered that substance use was higher in blue colour job workers. ${ }^{7}$ Mining is from one of those jobs where substance use is high. The present study identifies substance use to be two times greater in mineworkers than in non-mine workers. Moreover, a previous study also showed substance use to be higher among those who are more exposed to toxic dust material. ${ }^{36}$

An effective way to overcome substance use at the workplace may be to use a periodic awareness programme tailored to all villagers, especially targeting villages near the mining area. Self-help groups may be another beneficial way to increase awareness, particularly for those who are seeking help to quit and recover. Another method to control substance use could be to employ the district administration to intervene and keep a check on the availability of alcohol near the villages. A display of posters in clearly visible and prevalent areas, highlighting healthy alternatives may also be an efficient way to reduce substance abuse. $^{37}$

To the best of our knowledge, this is the first study measuring the prevalence of substance use among mineworkers in India. It would be interesting to assess the risk factors of substance use on the health of mineworkers; this could be an objective for a subsequent study, as it was out of the purview of this present research. The current study found a high prevalence of substance use among mineworkers compared to non-mine workers, attributed mainly to mining culture prevalent in the area. A significant weakness of this study is that the prevalence of substance uses and morbidity is based on a self-reported method of data acquisition, which is subject to knowledge and awareness of particular morbidities by the mineworkers and non-mine workers. Thus these findings may differ from the morbidity prevalence based on clinical test data and records of health care facilities. The current research uses a cross-sectional survey to collect data, which may underestimate the true prevalence of morbidities. Therefore, this study cannot be generalised, especially where health \& safety measures are more closely monitored.

\section{Conclusion}

This study is from one of a few studies that examine the demographic and substance use factors associated with mine workers. Results of this analysis highlight the high prevalence of substance use among mineworkers and provide insight into the demographic characteristics and behaviours associated with substance use. Khaini and bidi were the primary substances used by mineworkers. The prevalence of substances like khaini, bidi, alcohol, and gutkha was high when compared to nonmine workers. Substance use was prevalent among mineworkers and was a function of age at the onset of work.

\section{Role of the funding source}

This research did not receive any fund.

\section{Declaration of competing interest}

No conflict declared.

\section{Acknowledgments}

The suggestion and comments of anonymous reviewers of this journal greatly helped us to improve this manuscript. The authors would also like to thank all those who participated in the survey.

\section{References}

1. Bruno F. Drug and alcohol problems in the workplace in Italy. J Drug Issues. 1994:24:697-713.

2. Siegrist J, Rödel A. Work stress and health risk behavior. Scand J Work Environ Health 2006;32:473-481.

3. Siegrist J, Rodel A. Work stress and health risk behavior. Scand J Work Environ Health. 2006;32:473-481.

4. Petraitis J, Flay BR, Miller TQ. Reviewing theories of adolescent substance use: organizing pieces in the puzzle. Psychol Bull. 1995;117:67-86.

5. Pidd K, et al. Alcohol and Work: Patterns of Use, Workplace Culture and Safety. 2006 2006http://nceta.flinders.edu.au/files/1612/5548/2196/EN152.pdf.

6. Ames GM, Janes C. A cultural approach to conceptualizing alcohol and the workplace. Alcohol Health Res World. 1992;16:112-119.

7. Ames GM, Janes CR. Heavy and problem drinking in an American blue-collar population: implications for prevention. Soc Sci Med. 1987;25:949-960.

8. Ames GM, Grube JW. Alcohol availability and workplace drinking: mixed method analyses. J Stud Alcohol. 1999;60:383-393.

9. Schinder EO, Ruder AM. Epidemiology of coca and alcohol use among high-altitude miners in Argentina. Am J Ind Med. 1989;15:579-587.

10. Silva MC, Gaunekar G, Patel V, Kukalekar DS, Fernandes J. The prevalence and 
correlates of hazardous drinking in industrial Workers : a study from Goa , India. Alcohol Alcohol. 2003;38:79-83.

11. Bush DM, Lipari RN. Trends in heroin use in the United States: 2002 to 2013,Edited in substance use and substance use disorder by industry. The CBHSQ Report 1-18. 2015; 2015.

12. GOR. Mineral statistics. Department of Mines and Geology http://www.mines. rajasthan.gov.in/mineral-statistics.html.

13. Critchley JA, Unal B. Health effects associated with smokeless tobacco: a systematic review. Thorax. 2003;58:435-443.

14. Dutt B. Organizing the unorganized. http://re.indiaenvironmentportal.org.in/files/ minelabour-rajasthan.pdf; 2005.

15. NIMH. Detection of Silicosis Among Stone Mine Workers From Karauli District. National Institute of Miners Health; 2011.

16. Donoghue AM. Occupational health hazards in mining: an overview. Occup Med. 2004;54:283-289.

17. Ahmad A. Prevalence and predictors of occupational stress among quarry workers in rural Rajasthan, India. J Public Ment Health. 2017;16:132-143.

18. Adler NE, Newman K. Socioeconomic disparities in health: pathways and policies. Health Aff. 2002;21:60-76.

19. Solanki J, Gupta S, Chand S. Oral health of stone mine workers of Jodhpur city, Rajasthan, India. Saf Health Work. 2014;5:136-139.

20. Yadav S, Anand P, Singh H. Awareness and practices about silicosis among the sandstone quarry workers in desert ecology of Jodhpur, Rajasthan, India. J Hum Ecol. 2011;33:191-196

21. Mehta Rajesh. Silico-tuberculosis : burdening lives of miners a research study on prevalence and prevention of silico-tuberculosis in stone mines. www.gravis.org.in; 2010.

22. Gupta PC, Asma S. Bidi smoking and public health. http://mohfw.nic.in/ WriteReadData/1892s/file16-29724885.pdf; 2008.

23. Balasubramanian R. Prevalence of oral mucosal lesions among granite factory employees in Nanjangud Taluk, Mysore. Glob J Med Res. 2014;14.

24. TISS, GOI. Global Adult Tobacco Survey GATS 2 India 2016-17. 2017; 2017.
25. Abbas I, et al. Oral health status of underground coal mine workers of Ramakrishnapur, adilabad district, Telangana, India - a cross-sectional study. J Clin Diagn Res. 2016;10:28-31.

26. Dagli RJ, Kumar S, Dhanni C, Duraiswamy P, Kulkarni S. Dental health among green marble mine laborers, India. J Oral Health Community Dent. 2008;2:1-7.

27. Tiwari B. Impact of coal mining on Ecosystem health in Jaintia hills, Meghalaya. In: Ramakrishnana AN Purohit, Saxena KG, Rao KS, Maikhuri RK, eds. Conservation and Management of Biological Resources in Himalaya. Oxford and IBH Publlishing Co. Pvt. Ltd.; 1996.

28. Lehman WEK, Bennett JB. Job risk and employee substance Use : the influence of personal background and work environment factors. Am J Drug Alcohol Abuse. 2002;28:263-286.

29. Trice HM, Sonnenstuhl WJ. Drinking behavior and risk factors related to the work place: implications for research and prevention. J Appl Behav Sci. 1988;24:327-346.

30. Buchanan DJ. Studies on blood alcohol in the workers of A Zambian copper mine. Alcohol Alcohol. 1988;23:239-242.

31. De Matteis S, et al. Occupations associated with COPD risk in the large populationbased UK Biobank cohort study. Occup Environ Med. 2016;73 378 LP - 384

32. Saini G, Prabhat K, Gupta N. Drug addiction and periodontal diseases. J Indian Soc Periodontol. 2013;17:587.

33. Kandel DB, Logan JA. Patterns of drug use from adolescence to young Adulthood : 1 . Periods of risk for initiation, continued use, and discontinuation. Am J Public Health. 1984;74:660-666.

34. Jordan CJ, Andersen SL. Sensitive periods of substance abuse: early risk for the transition to dependence. Dev Cogn Neurosci. 2017;25:29-44.

35. Grant BF. Age at smoking onset and its association with alcohol consumption and DSM-IV alcohol abuse and dependence: results from the National Longitudinal Alcohol Epidemiologic Survey. J Subst Abus. 1998;10:59-73.

36. Graber JM, et al. High cigarette and poly-tobacco use among workers in a dusty industry: New Jersey quarry workers. J Occup Environ Med. 2016;58:1-18.

37. Malick R. Prevention of substance use disorders in the community and workplace. Indian J Psychiatr. 2018;60:559-563. 